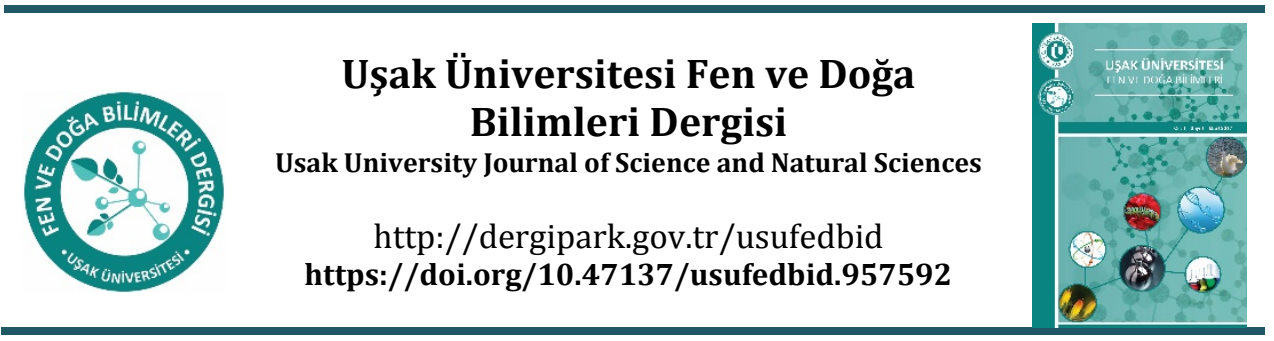

Araştırma Makalesi

\title{
Some Affine Surfaces in Simply Isotropic 3-Space
}

\author{
Gülsüm Solmaz ${ }^{1}$ Murat Kemal Karacan ${ }^{2 *}$ \\ ${ }^{1}$ Uşak University, Graduate Education Institute, Department of Mathematics, TURKEY \\ ${ }^{2} U$ şak University, Faculty of Science and Art, Department of Mathematics, TURKEY
}

Geliș: 25 Haziran 2021 Kabul: 22 Ağustos 2021/Received: 25 June 2020 Accepted: 22 August 2021

\begin{abstract}
In this paper, we have defined some of the special affine surfaces (Affine spheres, Affine ruled surfaces) and their dual surfaces given by the monge patch in Simply Isotropic 3-space. We also have given the Gaussian and the mean curvatures.
\end{abstract}

Keywords: Affine spheres, affine ruled surfaces, dual surfaces, simply isotropic 3-space.

\section{Özet}

Bu makalede, 3-boyutlu basit izotropik uzayda, Monge yamasi ile verilen bazı özel Afin yüzeyler ile (Afin küreler, Afin regle yüzeyler) bunların dual yüzeylerini tanımladık. Ayrıca bu yüzeylere ait Gauss ve ortalama eğriliklerini inceledik.

Anahtar Kelimeler: Afin küreler, afin regle yüzeyler, dual yüzeyler, basit izotropik 3-uzay.

(C)2021 Usak University all rights reserved.

\section{Introduction}

In mathematics, an affine space is a geometric structure that generalizes some of the properties of Euclidean spaces in such a way that these are independent of the concepts of distance and measure of angles, keeping only the properties related to parallelism and ratio of lengths for parallel line segments. In mathematics, and especially differential geometry, an affine sphere is a hypersurface for which the affine normals all intersect in a single point [5].The term affine sphere is used because they play an analogous role in affine differential geometry to that of ordinary spheres in Euclidean differential geometry. An affine sphere is called improper if all of the affine normals are constant.In that case, the intersection point mentioned above lies on the hyperplane at infinity [5]. Lone and Karacan studied the dual translation surfaces in three dimensional simply

\footnotetext{
**Corresponding author:

E-mail: murat.karacan@usak.edu.tr (ORCID ID: 0000-0002-2832-9444)
} 
isotropic space and they gave classification of dual translation surface with constant dual isotropic mean curvature or constant dual isotropic Guassian curvature [4]. Belkhelfa and Katou classified all affine semiparallel surfaces in $R^{3}$ with constant Pick invariant and they characterized affine semiparallel surfaces of constant Pick invariant but of which shape operators are not paralel [1]. Katou studied a new class of affine minimal hypersurfaces as higher dimensional analogues of affine minimal ruled surfaces [3]. Clelland and Miller investigated the geometric properties of hyperbolic affine flat affine minimal surfaces in the equiaffine space $A^{3}$ [2]. In this paper we have investigated the curvatures of affine spheres and affine ruled surfaces given by $[1,2,3]$ in simply isotropic 3-space.

\section{Preliminaries}

A simply isotropic space $I_{3}^{1}$ is a Cayley-Klein space defined from the three dimensional projective space $P\left(R^{3}\right)$ with the absolute figure which is an ordered triple $\left(w, f_{1}, f_{2}\right)$, where $w$ is a plane in $P\left(R^{3}\right)$ and $f_{1}, f_{2}$ are two complex-conjugate straight lines in $w$. The homogeneous coordinates in $P\left(R^{3}\right)$ are introduced in such a way that the absolute plane $w$ is given by $x_{0}=0$ and the absolute lines $f_{1}, f_{2}$ by $x_{0}=x_{1}+i x_{2}=0$, $x_{0}=x_{1}-i x_{2}=0$. The intersection point $F(0: 0: 0: 1)$ of these two lines is called the absolute point. The group of motions of the simply isotropic space is a six-parameter group given in the affine coordinates

by

$$
x=\frac{x_{1}}{x_{0}}, y=\frac{x_{2}}{x_{0}}, z=\frac{x_{3}}{x_{0}}
$$

$$
\begin{aligned}
& \bar{x}=a+x \cos \theta-y \sin \theta \\
& \bar{y}=b+x \sin \theta+y \cos \theta \\
& \bar{z}=c+c_{1} x+c_{2} y+z
\end{aligned}
$$

where $a, b, c, c_{1} C_{2}, \theta \in R$. Such affine transformations are called isotropic congruence transformations or $i$-motions [6]. Isotropic geometry has different types of lines and planes with respect to the absolute figure. A line is called non-isotropic (resp. completely isotropic) if its point at infinity does not coincide (coincides) with the point $F$. A plane is called non-isotropic (resp. isotropic) if its line at infinity does not contain $F$, otherwise. Completely isotropic lines and isotropic planes in this affine model appear as vertical, i.e, parallel to the $Z$-axis. Finally, the metric of the simply isotropic space $I_{3}^{1}$ is given by

$$
d s^{2}=d x^{2}+d y^{2} \text {. }
$$

A surface $S$ immersed in $I_{3}^{1}$ is called admissible if it has no isotropic tangent planes. For such a surface, the coefficients $E, F, G$ of its first fundamental form are calculated with respect to the induced metric and the coefficients $L, N, M$ of the second fundamental form, with respect to the normal vector field of a surface which is always completely isotropic. The (isotropic) Gaussian and (isotropic) mean curvature are defined by 


$$
K=k_{1} k_{2}=\frac{L N-M^{2}}{E G-F^{2}}, 2 H=k_{1}+k_{2}=\frac{E N-2 F M+G L}{E G-F^{2}}
$$

where $k_{1}, k_{2}$ are principal curvatures, i.e, extrema of the normal curvature determined by the normal section (in completely isotropic direction) of a surface. Since $E G-F^{2}>0$, for the function in the denominator we often put $W^{2}=E G-F^{2}$. The surface $S$ is said to be isotropic flat (resp. isotropic minimal) if $K$ (resp. $H$ ) vanishes [6].

We confine our discussion to regular surfaces $S$ without isotropic tangent planes. Thus, we may write in explicit form $z=f(u, v)$. A surface $S: z=f(u, v)$, seen as set of contact elements (points plus tangent planes) corresponds to a surface $S^{*}=\left(x^{*}(u, v), y^{*}(u, v), z^{*}(u, v)\right)$ parameterized by

$$
\begin{aligned}
& x^{*}=f_{u}(u, v) \\
& y^{*}=f_{v}(u, v) \\
& z^{*}=u f_{u}(u, v)+v f_{v}(u, v)-f(u, v)
\end{aligned}
$$

Contact elements along isotropic principal curvature lines of $S$ and $S^{*}$ correspond in the duality. Note that $S^{*}$ may have singularities which correspond to parabolic surface points of $S^{*}(K=0)$. This is reflected in the following relations between the isotropic curvature measures of dual surface pairs:

$$
K^{*}=\frac{1}{K}, H^{*}=\frac{H}{K},
$$

Thus, the dual surface to an isotropic minimal surface is also isotropic minimal [4].

\section{Some Affine Surfaces and Their Dual Surfaces}

In this section, we have investigated some of the special affine surfaces defined by $[1,2,3]$ and their dual surfaces given by the monge patch in Simply Isotropic 3-space. We will also give the Gaussian and the mean curvatures of these surfaces in Simply Isotropic 3space.

a) Type 1: If $z=u v+g(v)$, then the surface and its curvatures are given by

$$
\begin{gathered}
S_{1}(u, v)=(u, v, u v+g(v)) \\
K_{1}=-1, H_{1}=\frac{g^{\prime \prime}(v)}{2} .
\end{gathered}
$$

In this case, the dual surface and the curvatures are given by

$$
S_{1}^{*}(u, v)=\left(v, u+g^{\prime}(v),-g(v)+v\left(u+g^{\prime}(v)\right)\right)
$$




$$
K_{1}^{*}=-1, H_{1}^{*}=-\frac{g^{\prime \prime}(v)}{2}
$$

So, we have following results.

Corollary 3.1. There are no isotropic flat surfaces $S_{1}$ and $S_{1}^{*}$.

Theorem 3.1. Let $S_{1}$ and $S_{1}^{*}$ be a surface of Type 1 and a dual surface of Type 1 in $I_{3}^{1}$. If the surfaces $S_{1}$ and $S_{1}^{*}$ are isotropic minimal then we have $g(v)=c_{1} v+c_{2}, c_{1}, C_{2} \in R$.

Proof: Suppose the mean and dual mean curvatures $H_{1}$ and $H_{1}^{*}$ of Type 1 in $I_{3}^{1}$ vanishes identically, from (1) and (2) we have $g^{\prime \prime}(v)=0$ and $g(v)=c_{1} v+c_{2}, c_{1}, c_{2} \in R$.

Now, we consider dual translation surface of Type 1 with non-zero dual constant mean curvature in $I_{3}^{1}$. If the mean curvatures $H_{1}$ and $H_{1}^{*}$ are non-zero constant, from (1) and (2), we have $g^{\prime \prime}(v)-2 H_{1}=0$ and $g^{\prime \prime}(v)+2 H_{1}^{*}=0$. So we get

$$
\begin{aligned}
& g(v)=H_{1} v^{2}+c_{1} v+c_{2}, c_{1}, c_{2} \in R, \\
& g(v)=-H_{1}^{*} v^{2}+c_{1} v+c_{2}, c_{1}, c_{2} \in R .
\end{aligned}
$$

Corollary 3.2. Let $S_{1}$ and $S_{1}^{*}$ be a surface of Type 1 and a dual surface of Type 1 in $I_{3}^{1}$. If the surfaces $S_{1}$ and $S_{1}^{*}$ have the constant mean curvatures, then the function $g(v)$ is given by (3).

b) Type 2: If $z=v \tan u+f(u)$, then the surface and its curvatures are given by

$$
\begin{gathered}
S_{2}(u, v)=(u, v, v \tan u+f(u)) \\
K_{2}=-\frac{1}{\cos ^{4} u}, H_{2}=v \frac{\sin u}{\cos ^{3} u}+\frac{f^{\prime \prime}(u)}{2} .
\end{gathered}
$$

In this case, the dual surface and the curvatures are given by

$$
\begin{aligned}
& S_{2}^{*}(u, v)=\left(\frac{v}{\cos ^{2} u}+f^{\prime}(u), \tan u,-f(u)+u\left(\frac{v}{\cos ^{2} u}+f^{\prime}(u)\right)\right) \\
& K_{2}^{*}=-\cos ^{4} u, H_{2}^{*}=-\frac{\cos u}{2}\left(2 v \sin u+\cos ^{3} f^{\prime \prime}(u)\right) .
\end{aligned}
$$

So, we have following result.

Corollary 3.3. There are no isotropic flat and isotropic minimal surfaces $S_{2}$ and $S_{2}^{*}$. 
c) Type 3: If $z=\frac{v^{2}}{2}+f(u)$, then the surface and its curvatures are given by

$$
\begin{gathered}
S_{3}(u, v)=\left(u, v, \frac{v^{2}}{2}+f(u)\right) \\
K_{3}=f^{\prime \prime}(u), H_{3}=\frac{1}{2}\left(1+f^{\prime \prime}(u)\right) .
\end{gathered}
$$

In this case, the dual surface and the curvatures are given by

$$
\begin{gathered}
S_{3}^{*}(u, v)=\left(f^{\prime}(u), v, \frac{v^{2}}{2}-f(u)+u f^{\prime}(u)\right) \\
K_{3}^{*}=\frac{1}{f^{\prime \prime}(u)}, H_{3}^{*}=\frac{1}{2}\left(1+\frac{1}{f^{\prime \prime}(u)}\right) .
\end{gathered}
$$

Now, we consider the surface of Type 3 and its dual surface. If the Gaussian curvatures $K_{3}$ and $K_{3}^{*}$ are non-zero constant, from (6) and (7), we have $f^{\prime \prime}(u)-K_{3}=0$ and $f^{\prime \prime}(u) K_{3}^{*}-1=0$. So we get

$$
\begin{aligned}
& f(u)=K_{3} \frac{u^{2}}{2}+c_{1} u+c_{2}, c_{1}, c_{2} \in R, \\
& f(u)=\frac{u^{2}}{2 K_{3}^{*}}+c_{1} u+c_{2}, c_{1}, c_{2} \in R .
\end{aligned}
$$

So, we have the following theorem.

Theorem 3.2. Let $S_{3}$ and $S_{3}^{*}$ be a surface of Type 3 and a dual surface of Type 3 in $I_{3}^{1}$. If the surfaces $S_{3}$ and $S_{3}^{*}$ have constant the Gaussian curvatures, then the function $f(u)$ is given by (8).

Now, we consider the surface and its dual surface. If the mean curvatures $H_{3}$ and $H_{3}^{*}$ are non-zero constant, from (6) and (7), then we have

$$
\begin{aligned}
& f(u)=\frac{1}{2}\left(-1+2 H_{3}\right) u^{2}+c_{1} u+c_{2}, c_{1}, c_{2} \in R, \\
& f(u)=\frac{u^{2}}{2\left(-1+2 H_{3}^{*}\right)}+c_{1} u+c_{2}, c_{1}, c_{2} \in R .
\end{aligned}
$$


Corollary 3.4. Let $S_{3}$ and $S_{3}^{*}$ be a surface of Type 3 and a dual surface of Type 3 in $I_{3}^{1}$. If the surfaces $S_{3}$ and $S_{3}^{*}$ have constant the mean curvatures, then the function $f(u)$ is given by (9).

d) Type 4: If $z=v e^{u}+f(u)$, then the surface and its curvatures are given by

$$
\begin{aligned}
& S_{4}(u, v)=\left(u, v, v e^{u}+f(u)\right) \\
& K_{4}=-e^{2 u}, H_{4}=\frac{1}{2}\left(v e^{u}+f^{\prime \prime}(u)\right) .
\end{aligned}
$$

In this case, the dual surface and the curvatures are given by

$$
\begin{aligned}
& S_{4}^{*}(u, v)=\left(v e^{u}+f^{\prime}(u), e^{u},-f(u)+u\left(v e^{u}+f^{\prime}(u)\right)\right) \\
& K_{4}^{*}=-e^{-2 u}, H_{4}^{*}=-\frac{e^{-2 u}}{2}\left(v e^{u}+f^{\prime \prime}(u)\right) .
\end{aligned}
$$

Corollary 3.5. There are no isotropic flat and isotropic minimal surfaces $S_{4}$ and $S_{4}^{*}$.

e) Type 5: If $z=v u+f(v)+v g(v)$, then the surface and its curvatures are given by

$$
\begin{gathered}
S_{5}(u, v)=(u, v, v u+f(v)+v g(v)) \\
K_{5}=-1, H_{5}=\frac{1}{2}\left(2 g^{\prime}(v)+f^{\prime \prime}(v)+v g^{\prime \prime}(v)\right) .
\end{gathered}
$$

In this case, the dual surface and the curvatures are given by

$$
\begin{gathered}
S_{5}^{*}(u, v)=\left(v, u+g(v)+f^{\prime}(v)+v g^{\prime}(v),-f(v)-v g(v)+v\left(u+g(v)+v g^{\prime}(v)\right)\right) \\
K_{5}^{*}=-1, H_{5}^{*}=\frac{1}{2}\left(-2 g^{\prime}(v)-f^{\prime \prime}(v)-v g^{\prime \prime}(v)\right) .
\end{gathered}
$$

Suppose the mean and dual the mean curvatures $H_{5}$ and $H_{5}^{*}$ of Type 5 in $I_{3}^{1}$ vanishes identically, from (12) and (13), we have

or

$$
g(v)=-\frac{f(v)}{v}-\frac{c_{1}}{v}+c_{2}, c_{1}, c_{2} \in R
$$

$$
f(v)=-v g(v)+c_{1} v+c_{2}, c_{1}, c_{2} \in R
$$

Corollary 3.6. There are no isotropic flat surfaces $S_{5}$ and $S_{5}^{*}$. 
Corollary 3.7. If the surfaces $S_{5}$ and $S_{5}^{*}$ are isotropic minimal, then the functions $f$ and $g$ are related to each other.

\section{References}

1. Belkhelfa M, Katou M. Affine semiparallel surfaces with constant Pick invariant. Hokkaido Mathematical Journal, 2005; 34: 355-374.

2. Miller JM, Clelland JN. A characterization of hyperbolic affine flat affine minimal surfaces in $A^{3}$. Differential Geometry and its Applications, 2016; 36: 134-148.

3. Katou M. New affine minimal ruled hypersurfaces, J. Math. Soc. Japan, 2006; 58(3): 869-883.

4. Lone MS, Karacan MK. Dual Translation Surfaces in the three Dimensional Simply Isotropic Space $I_{3}^{1}$. Tamkang Journal of Mathematics, 2018; 49(1): 6777.

5. Shikin EV. Affine sphere, Encyclopedia of Mathematics, EMS Press; 1994.

6. Sipus ZM. Translation surfaces of constant curvatures in a simply isotropic space. Period Math Hung, 2014; 68: 160-175. 